Asian-Australasian Journal of

Bioscience and Biotechnology

ISSN 2414-1283 (Print) 2414-6293 (Online)

www.ebupress.com/journal/aajbb

\title{
Article \\ Landing and distribution of captured fish in selected landing centers in Kishoreganj haor, Bangladesh
}

Alam AKM Nowsad, Mousumi Akter ${ }^{*}$ and Al-Shahriar

Department of Fisheries Technology, Faculty of Fisheries, Bangladesh Agricultural University, Mymensingh2202, Bangladesh

*Corresponding author: Mousumi Akter, Department of Fisheries Technology, Faculty of Fisheries, Bangladesh Agricultural University, Mymensingh-2202, Bangladesh. E-mail: hmakterbau@gmail.com

Received: 25 June 2021/Accepted: 23 August 2021/ Published: 31 August 2021

\begin{abstract}
The present study illustrates species diversity, landing quality and catch composition of fish in 10 fish landing centers of 5 haor upazilas of Kishoreganj haor for 12 months from January 2018 to December 2018. A total of 15 different types of fishing gears were detected to harvest fish in different stations throughout a year. Most of the fishing (46.88\%) was conducted by small groups of $2-5$ fishers, while $24.87 \%$ of the fishing was conducted by $6-10$ fishers. The average depth of water in haor associated rivers and inundated lands during monsoon period varied from $4.87 \pm 0.99$ to $15.2 \pm 6.71$ and $2.6 \pm 0.55$ to $6.2 \pm 2.68$ feet respectively. Fifty different types of fish species were landed in the selected 10 landing centers, where both cultured fish and captured fish were present. Fishes under the Cyprinidae family included 9 species, viz. Labeo rohita. Gebelion catla, Cirrhinus cirrhosus, Labeo calbasu, Hypophthalmichthys nobilis, Hypophthalmichthys molitrix, Cyprinus carpio, Ctenopharyngodon idella, Labeo gonius covered a maximum of $12450.61 \pm 468.32$ MT. The second largest landed fish under the family Schilbeidae included 4 species Silonia silondia, Mystus armatus, Mystus cavasius Pseudeutropius atherinoides. Other fish species landed were identified as: 4 species under the family Mastacembelidae, 2 species under Siluridae, 3 species under Clupidae, 1 species under Ambassidae, 2 species under Palaemonidae, 3 species under Channidae, 1 species under Gobidae, 2 species under Bagridae and 1 species from each of the family Heteropneustidae, Pangasiidae, Clariidae, Ailiidae, Botiidae, Nandidae, Cichlidae, Anabantidae, Osphronemidae, Ambassidae, Notopteridae, Notopteridae,Belonidae and Gobiidae. Highest number 14 species landed were under catfish category, followed by 9 species of major carp. The captured total fish landed in 10 landing centers in 12 month period accounted 12,574 \pm 1029.64 MT, but the cumulative total landed fish, including pond fish, was 15,795 $\pm 1666061.93 \mathrm{MT}$, as calculated by 45 Aratdars of 10 landing centers. About $88.35 \%$ fishermen sold the captured fish at local arat, but the rest $(11.65 \%)$ were sold to large traders (paiker), retailers and consumers. A 56.6\% of the fishermen cleaned the captured fish by river water, while $43.4 \%$ fishermen did not use water for washing fish before selling. After harvest, $45.62 \%$ fishers used clean utensils for carrying fish to arat and 54.38\% used uncleaned utensils. Average harvest per fishermen per day was $23.9 \mathrm{~kg}$, of which $22.26 \mathrm{~kg}$ was sold in the market and $1.64 \mathrm{~kg}$ was used for own family consumption. Out of total fish landed, a $45.2 \%$ was transported by fish hold of the country boat, $21.9 \%$ by bamboo basket and $14.8 \%$ by plastic crate. Relatively small quantity of fish was transported by insulated ice box (4.7\%), aluminum container (2.7\%) and gunny bag (3.2\%). A 32.6\% fisherman used ice and $67.4 \%$ fishermen did not use ice after harvesting of fish. During fishing season, fish and ice ratio used by the transporters was 2.62:1. The highest number of auctioneers were found in Chamra Ghat of Karimganj, while lowest were in Nikli, with an area of $55 \pm 1.44$ and $25 \pm 8.42$ decimal area per auctioneers being used for fish business.
\end{abstract}

Keywords: Kishoreganj haor; fish landing centers; fish biodiversity; fish handling; fish quality 


\section{Introduction}

Bangladesh is enriched with wide variety of huge open water bodies like numerous rivers, canal, beels, lakes and vast area of floodplains. Total area of the inland water body of our county is about 4.7 million hectors where inland open water body is about 3.9 million hectors including river and estuaries, sundarban, beel, kaptai lake and floodplain. In 2018-19 fiscal year, total fish production was 43.84 lakh MT. Inland open water fish production was 12.35 lakh MT of which floodplain fish production was 7.8 lakh MT which is $17.82 \%$ of total fish production (DoF, 2020). The haor basins are mainly located in the North-eastern region of Bangladesh covering about 1.99 million hectares of area (Alam et al., 2010; CEGIS, 2012; Nowreen et al., 2014) and accommodating about 19.37 million people (CEGIS, 2012). It covers more than $24,000 \mathrm{~km}^{2}$ area, approximately $17 \%$ of the country's land area (Alam and Hasan, 2010) and stretches over parts of seven districts of the Northeastern region of Bangladesh, i.e. Sylhet, Sunamganj, Habiganj, Moulvibazar, Netrokona, Brahmanbaria and Kishoreganj (MoWR, 2010), though it does not have any defined territory. There are about 373 haors located in the districts of Sunamganj, Sylhet, Maulvibazar, Habiganj, Brahmanbaria, Netrokona and Kishoreganj, and covering an area of about 858,000 ha, which is around $43 \%$ of total area of the haor region (Master Plan of Haor Areas, 2012). Beel and haors covers an area 1.14 lakh ha and inundable floodplains 54.86 lakh ha (Ali, 1997) A haor is a back swamp or bowl shaped depression, between the natural levees of a river. Haors are flooded annually during the rains and they remain under water for several months of the year. When they drain out during the post-monsoon months, some permanent water bodies, or beels remain in the deepest parts of the haors (IUCN - Bangladesh, 2004). The haors of Kishoreganj covers an area of 121,590 ha perennial watershed. Although fisheries plays significant role in the economy of the country, haor fisheries is being faced threat to extinction. The main causes of declining of fisheries are the degradation of haor ecosystem, over exploitation, pollution and injudicious intrusion of human. Besides, lack of awareness about the sustainable management of haor water or open water fisheries convert this poor situation into worst. The multiplicity of traditional fishing, and/or illegal fishing practice and inappropriate post-harvest fishery management, along with socio-economic condition, geographical position, carelessness and lack of technical skill are marginalizing the livelihood of haor fishery communities, which is further being triggered by poor. It is therefore, almost certain than haor fishery communities are completely unorganized and fishery harvest and post-harvest management is inferior. The poverty of the communities is the cause and effect of bewildering fisheries of haor (Nowsad, 2019). Haor fishes are marketed throughout the country through 24 fish landing centers and about 200 aratdars in Kishorganj. In addition, landed fishes are locally sold by 220 fish markets scattered throughout the haor upzilas (DoF, 2015). The production of capture fisheries depends heavily on different open water resources such as rivers, beels, floodplains, and haors. In Bangladesh, haors contribute approximately $10 \%$ of the total capture fisheries production (DoF, 2018), however this amount has reduced over time. Fish and fisheries sector plays an important role in the national economy of the country. Diversity of fishes meet nutritional requirement and ecosystem balance. In the haor area of Kishorganj district, a total of 46 fish species were identified under 17 families and most of the fish species (15 species) belong to Cyprinidae family followed by Bagaridae (4), Channidae (4), Schilbeidae (3), Mastacembelidae (3), Centropomidae (3), Siluridae (2), Anabantidae (2), Palaemonidae (2) and others (8) (Hasan et al., 2017).

Haor contains diversified flora and fauna especially reptiles, birds, fish species, amphibians etc., which play an important role in the existence of haor ecosystem (Choudhury, 2016). According to Pandit et al., 2015 local fishermen commonly caught over 84 species of fish in the haor region. Islam et al., 2008 recorded 108 species under 29 families of 10 orders from the haor region. Hence, the haor region has significant roles for fish production, maintaining biodiversity, meeting local and regional demand (Salauddin and Islam, 2011). However, the Kishoreganj haor region is famous for its richest SIS (Small Indigenous Fish Species) biodiversity, consisting of 30 species belonging to 7 orders and 15 families (Rownok et al., 2014). Kishoreganj is a low-lying 'Vati' district, characterized with inundation of 110,882 ha land as haor by flood water for 4-6 months. The district harvests highly popular "haor-fish" of about 50,600 MT per annum, with 20,000 fishers and traders involved in fishery business (DoF, 2012). Being wild harvest, the "haor-fish" are considered to be tastiest fish and highly relished by the affluent consumers in metropolitan cities country-wide (Nowsad, 2021). However, post-harvest handling situation of landed fish in Kishoreganj haor area is completely unknown, since no study has so far been conducted on post-harvest condition of valuable haor fishes. Because of remote and isolated locations of haor and limited access to government support services and GO/NGO extension facilities, haor areas of the country remain neglected in many cases, including fishing, fish landing, handling, marketing and distribution (Nowsad, 2016). Moreover, very limited research has been done on the species diversity, landing pattern and quality of fish in the floodplain areas except that of Nowsad et al., 2021a, 2021b, and 2021c. 
Therefore, the present study was conducted to assess the fish species diversity, landing pattern and quality and catch composition in 10 selected landing spots of Kishoreganj haor.

\section{Materials and Methods}

\subsection{Study area and duration}

The study was conducted at 10 fish landing spots in 5 haor Upazilas in Kishoreganj district (Figure 1). These were Chamra ghat matshya arat, Karimganj mach bazar and Morichkhali matshya arat in Karimganj upazila; Tarail matshya arat in Tarail upazila; Chouganga matshya arat in Itna upazila; Korgaon bazar matshya arat, Kotiadi matshya arat and Dhuldia matshya arat in Kotiadi upazila; Rodar podda matshya arat and Nikli notun bazar matshya arat in Nikli upazila. The study was performed for 12 months, from January 2018 to December, 2018.

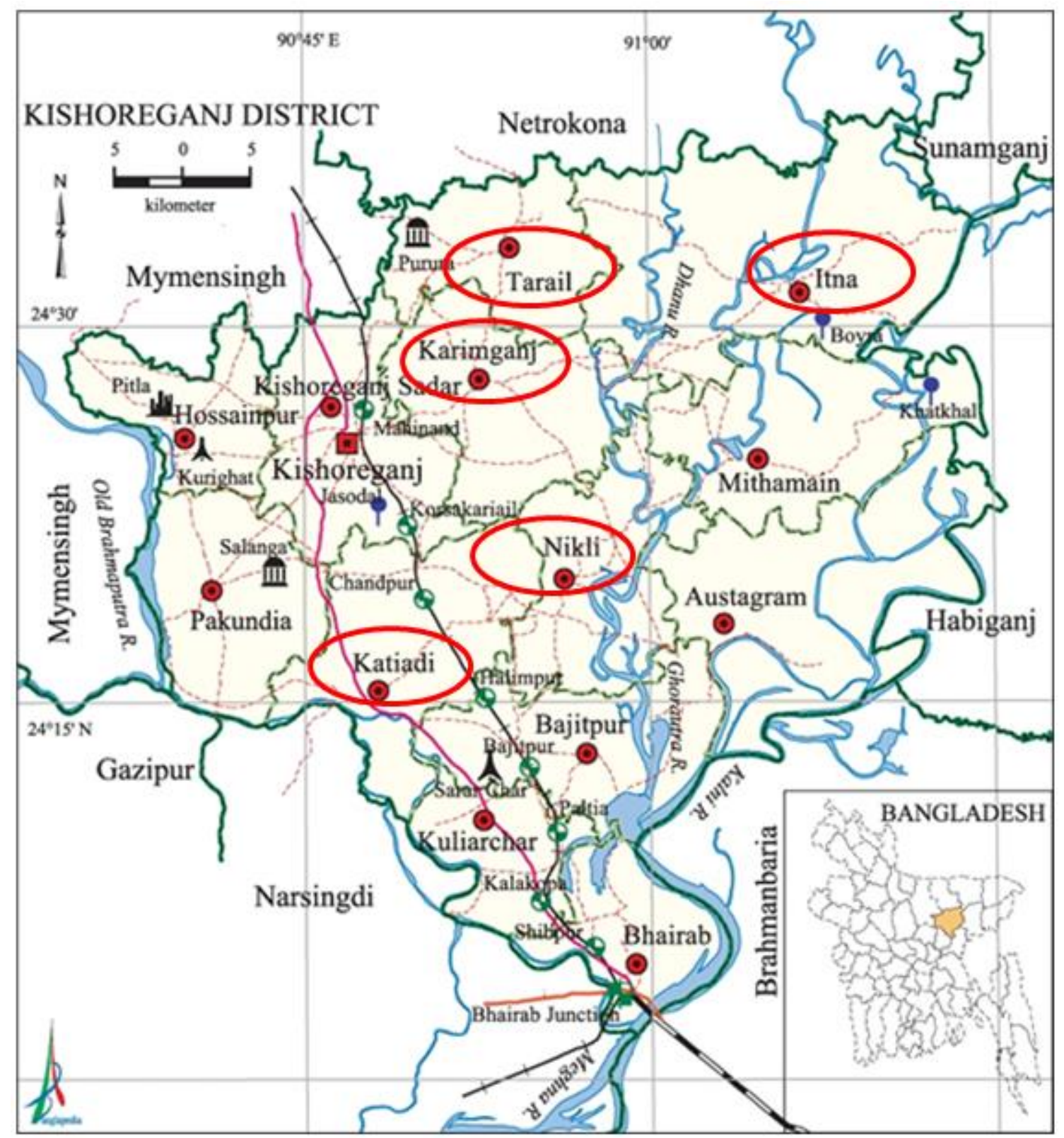

Figure 1. Map for showing the location of study area. 


\subsection{Method of data collection}

The study was based on the collection of primary data. From the selected study area, primary data were collected from 252 stakeholders through questioner interview, focus group discussion with intermediaries, cross-check interview with key informants and direct catch data from landing centers in 2018. A structured questioner form was developed by rigorous drafting, editing and field validating by the community. Primary data were validated by the secondary data collected from respective Upazila fisheries offices and District fisheries office of Kishoreganj.

\subsection{Data Analysis}

All the collected data were accumulated in Excel sheet and analyzed by Microsoft Excel 2016 and then presented in textual, tabular and graphical forms for easy understanding of present findings.

\section{Results and Discussion}

\subsection{Status of gear used in Kishorgonj haor}

A variety of fishing gears were used in Kishoreganj haor. A total of 15 different types of fishing gears were detected to harvest fish in different stations throughout a month (Table 1). Most fishing is conducted in small groups of $2-5$ fishers (46.88\%) in Kishoreganj haor with the $24.87 \%$ fishing is conducted by $6-10$ fishers. Fishing as a part of single person is conducted by $22.28 \%$ and a small portion of fishing is conducted by larger groups (5.97\%). Majority of the fishing is done by small group of fishers in all fish landing centers except Tarial and Chauganga. In Tarial and Morichkhali most of the fishing is conducted by single person and in Kotiadi and Chauganga above 50\% fishing is done by medium group fishers (Figure 2). There are no such types of published report, so it is not possible to compare the present findings. Sayeed et al., 2015 stated that 15 types of fishing gears of five major groups such as nets, traps, hooks and line, wounding gears and fish aggregating devices were operated for fishing by fishermen in Hakaluki haor, Moulvibazar. Rahman and Akhter, 2015 stated that a total of 34 different types of fishing gears where seine net, gill net, lift net, push net, cast net, fish trap and hooks and lines were 12 (34\%), 4 (12\%), 2 (6\%), 4 (12\%), 1 (3\%), $7(21 \%)$ and $4(12 \%)$, respectively were used to catch fish from haors. Sayeed, 2010 reported that thirty four different gears in six categories were recorded in Chalan beel in greater Pabna and Natore districts. Holder, 2002 noted 9 types of gear, were classified into three groups as nets, traps and wounding gears, used in two beels (Doba and Chara) of Mymensingh sadar upazila, Mymensingh. BCAS, 1991 estimated about 30 different types of gears used by fishermen in Halti beel in Natore and Rajshahi districts.

Table 1. Different types of fishing gears used in Kishoreganj haor.

\begin{tabular}{|l|l|l|l|l|l|}
\hline Sl no. & Name of gear & No. of gear used & Sl no. & Name of gear & No. of gear used \\
\hline 1 & Kona ber jal & 86 & 9 & Borshi & 399 \\
\hline 2 & Pai ber jal & 23 & 10 & Kora jal & 152 \\
\hline 3 & Chonda ber jal & 34 & 11 & Chunga & 80 \\
\hline 4 & Gorgoria jal & 87 & 12 & Bandor jal & 8 \\
\hline 5 & Chap jal & 64 & 13 & Chai & 33 \\
\hline 6 & Vim jal & 47 & 14 & Ber jal & 10 \\
\hline 7 & Jhaki jal & 207 & 15 & Dak jal & 97 \\
\hline 8 & Thela jal & 142 & & & \\
\cline { 1 - 3 } & & & &
\end{tabular}




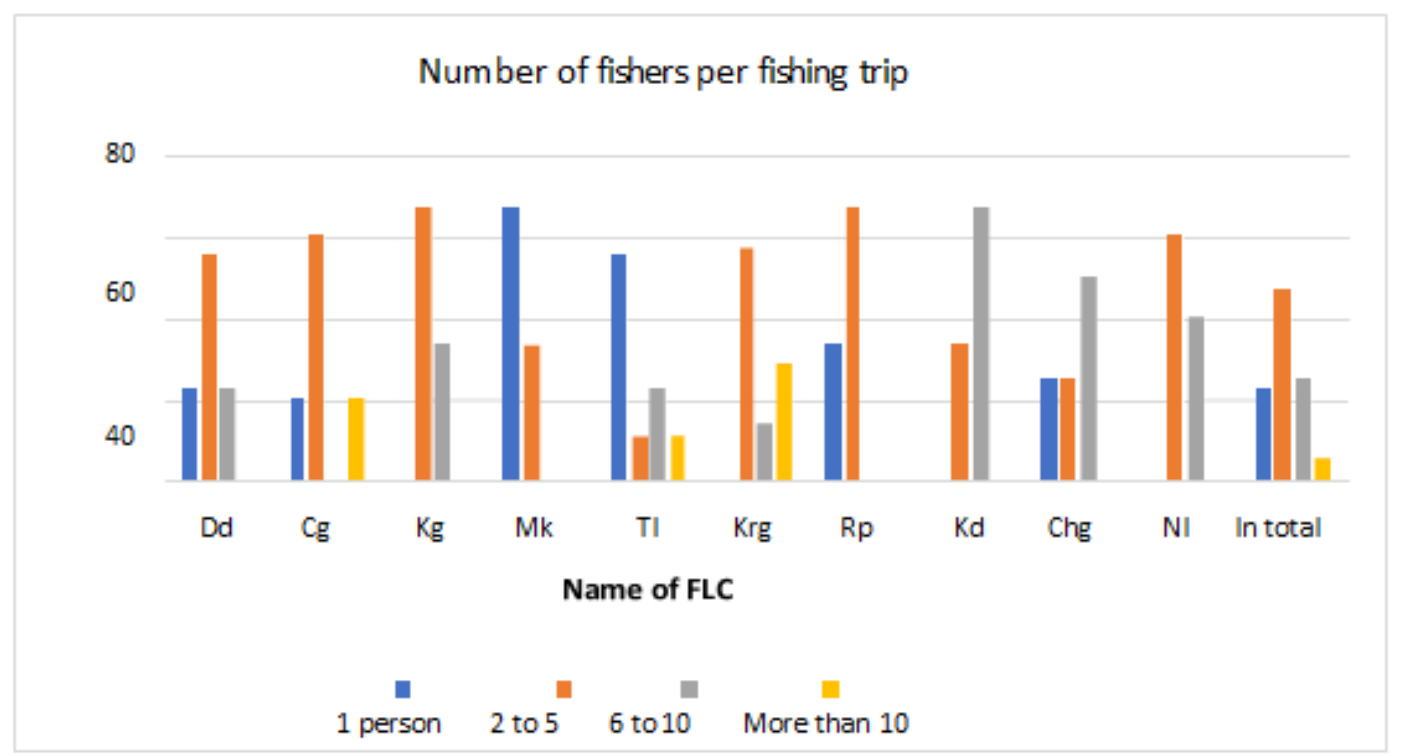

Figure 2. Number of fishermen per fishing trip in Kishoreganj haor basin.

\subsection{Water Depth of the haor}

The Water depth of river and land in Kishoreganj haor have been summarized in Figure 3 and 4 respectively. The average depth of water in river varied from $4.87 \pm 0.99$ to $15.2 \pm 6.71$ feet. The minimum depth of water varied from $4.87 \pm 0.99$ feet in February to $6.9 \pm 4.45$ feet in July-November. The maximum depth of water varied from 12.12 \pm 0.78 feet in February to $15.2 \pm 6.71$ feet in July-November (Figure 3). The average depth of water in land varied from $2.6 \pm 0.55$ to $6.2 \pm 2.68$ feet. The minimum depth of water varied from $2.6 \pm 0.55$ feet to $4.0 \pm 2.27$ feet. The maximum depth of water varied from $4.8 \pm 0.45$ feet to $6.2 \pm 2.68$ feet (Figure 4 ). Salauddin and Islam, 2011 found that the water body in haor area remains at the zero level from January to March and then starts to increase and again go down from August. Mostly, in June and July, the water level reaches the highest point. According to NERP, 1995, almost all haor basins' are inundated for 7-8 months to depths of $5 \mathrm{~m}$ or more during the monsoon. This finding are more or less comply with present findings.

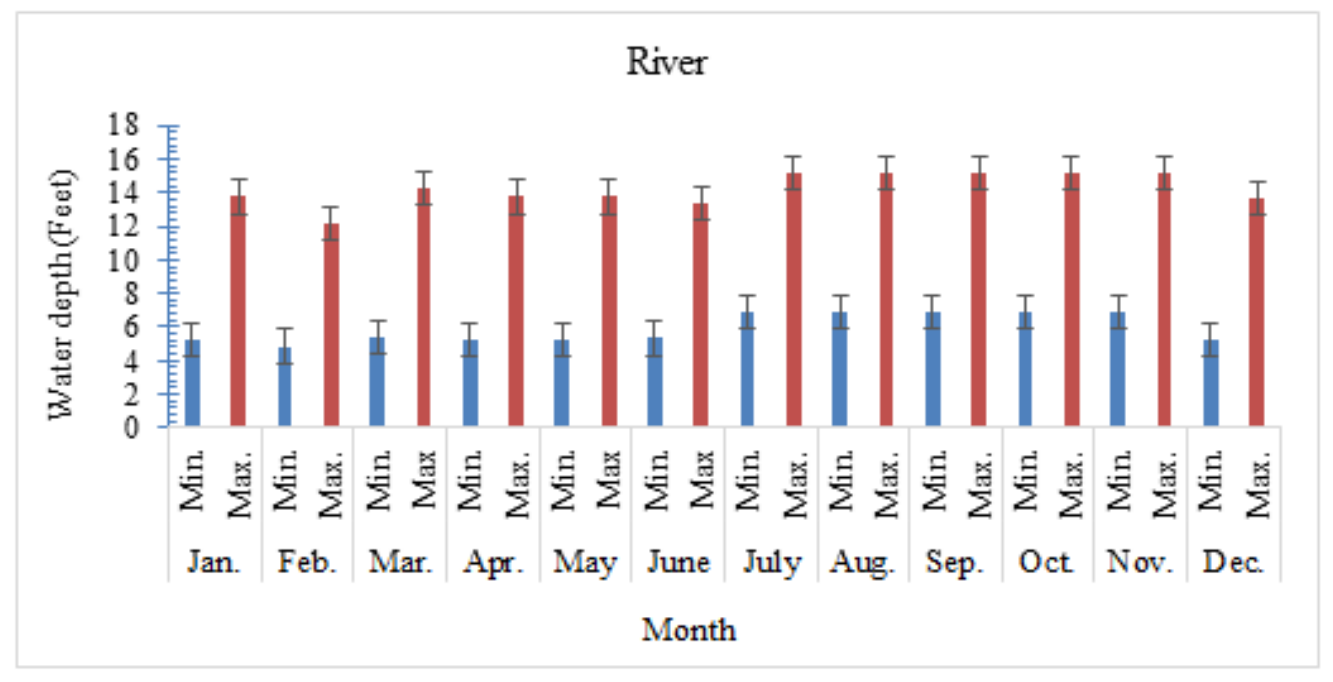

Figure 3. Water depth of river (feet) in Kishoreganj haor. 


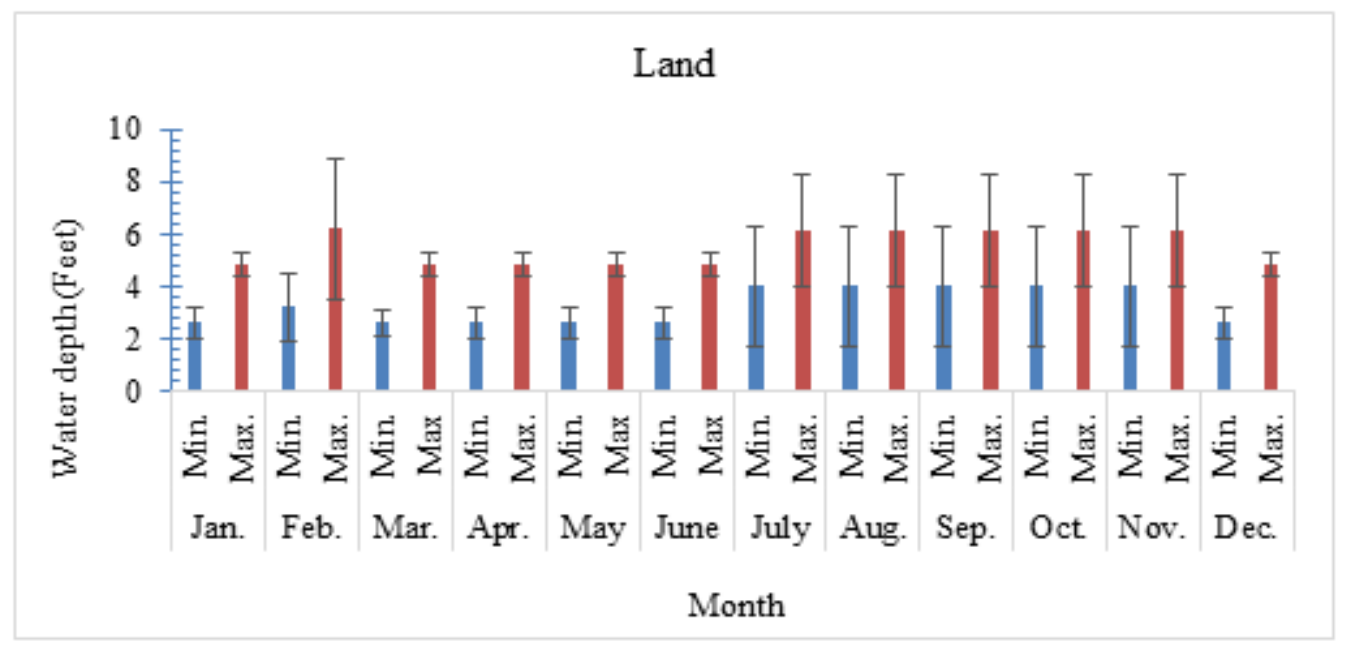

Figure 4. Water depth of land during monsoon (feet) in Kishoreganj haor.

\subsection{Species diversity of fish in the study area}

About 50 different types of fish species were recorded in selected haor area on the basis of the landed fish in the selected landing centers (Table 2). Table 2 shows the species wise and family wise landed fish (Metric ton) in different landing centers. Fishes under the Cyprinidae family included 9 species, viz. Labeo rohita. Gebelion catla, Cirrhinus cirrhosus, Labeo calbasu, Hypophthalmichthys nobilis, Hypophthalmichthys molitrix, Cyprinus carpio, Ctenopharyngodon idella, Labeo gonius covered a maximum of $12450.61 \pm 468.32$ MT. The second largest landed fish under the family Schilbeidae included 4 species Silonia silondia, Mystus armatus, Mystus cavasius Pseudeutropius atherinoides. Other fish species landed were identified as: 4 species under the family Mastacembelidae, 2 species under Siluridae, 3 species under Clupidae, 1 species under Ambassidae, 2 species under Palaemonidae, 3 species under Channidae, 1 species under Gobidae, 2 species under Bagridae and 1 species from each of the family Heteropneustidae, Pangasiidae, Clariidae, Ailiidae, Botiidae, Nandidae, Cichlidae, Anabantidae, Osphronemidae, Ambassidae, Notopteridae, Notopteridae,Belonidae and Gobiidae. Highest number 14 species landed were under catfish category, followed by 9 species of carps. Suravi et al., 2017 studied fish diversity of Dekar haor in Sunamganj, where they found 8 species of carps, 4 snakeheads, 3 eels, 11 catfishes and 1 minnow. More or less similar species diversities were also recorded in our study. Islam et al., 2020 recorded a total of 79 species of fishes belonging to 27 families under 9 orders in kishoreganj haor on the basis of direct observation and using morphometric and meristic characteristics which is higher than present findings. The highest number of species (10) found under the family Cyprinidae and major carps and exotic carps were the highest in the Daudkandi floodplain fisheries (DFPF) landings (Nowsad, 2020).

\subsection{Landing of fish in the study area}

Trends and frequency of catch and landing of fish in 10 landing centers in Kishoreganj haor area have been presented in Tables 3 and 4. Fishermen generally catch fish in different natural water bodies including haor waters and rivers. The cumulative catch by the fishermen involved with the 10 landing centers accounts $12,574 \pm 1029.64$ MT each year (Table 2). Quantity of landed fish in the landing centers are obviously different from that of the fishermen's catch, because the quantity of landed fish not only depend on wild harvest, but a significant portion also comes from the culture ponds or outsides. This phenomenon agrees well with the existing scenario since the total landed fish in the FLCs per annum was accounted to be $15,795 \pm 1666061.93$ MT, as calculated by 45 Aratdars of 10 landing centers (Table 4). There is no government statistics on upazilabased or haor-based landed and harvested fish in Kishoreganj or elsewhere in Bangladesh. So, data could not be verified with any independent sources. This fishery statistical data was directly collected from the landing stations in Kishoreganj haor. According to the Fisheries Statistics of Bangladesh, 2018-19, total fish production in Kishoregonj district was 76410 MT where river, beel and floodplain production were 2403, 6781 and 42094 MT respectively which was much higher than our findings. Chandra et al., 2010 noted that floodplain productivity tended to be somewhat higher and, in some cases, had been high as $6000 \mathrm{~kg} / \mathrm{ha}$. Highest catch was recorded in winter season (November to February) accounted 9804.78 MT, 8964.99 MT in pre-winter season (August to October) and 7865.31 MT in monsoon period (March to July) (Figure 5). 
Table 2. Landing center-wise species composition of landed fish in Kishoreganj haor (Metric ton).

\begin{tabular}{|c|c|c|c|c|c|c|c|c|c|c|c|c|c|c|c|}
\hline Category & Local Name & Scientific name & Family name & $\mathrm{Tl}$ & Kd & Krg & Kg & Dd & $\mathrm{Cg}$ & Mk & Chg & $\mathbf{N I}$ & Rp & \begin{tabular}{|l|}
$\begin{array}{l}\text { Total (Species } \\
\text { wise) }\end{array}$ \\
|
\end{tabular} & $\begin{array}{l}\begin{array}{l}\text { Total (Family } \\
\text { wise) }\end{array} \\
\end{array}$ \\
\hline \multirow[t]{9}{*}{ Carps } & Rui & Labeo rohita & Cyprinidae & $103.10 \pm 5.21$ & $132.18 \pm 7.68$ & $92.48 \pm 5.76$ & $54.14 \pm 5.67$ & $154.02 \pm 7.34$ & $116.93 \pm 6.43$ & $19.92 \pm 3.56$ & $255.64 \pm 13.65$ & $24.78 \pm 3.21$ & $25.18 \pm 3.54$ & $978.38 \pm 73.22$ & \multirow{13}{*}{$12450.61 \pm 468.32$} \\
\hline & Catla & Gebelion catla & & $54.45 \pm 2.14$ & $82.36 \pm 5.24$ & $70.68 \pm 4.53$ & $40.37 \pm 4.53$ & $103.88 \pm 6.54$ & $77.70 \pm 3.46$ & $16.36 \pm 3.76$ & $202.24 \pm 14.54$ & $17.65 \pm 1.76$ & $21.56 \pm 2.76$ & $687.25 \pm 55.69$ & \\
\hline & Mrigal & Cirrhinus cirrhosus & & $132.43 \pm 5.98$ & $98.63 \pm 5.98$ & $77.00 \pm 6.85$ & $61.03 \pm 6.34$ & $126.19 \pm 5.45$ & $100.49 \pm 6.43$ & $20.78 \pm 4.76$ & $249.18 \pm 10.23$ & $17.21 \pm 2.76$ & $27.87 \pm 4.32$ & $910.80 \pm 69.58$ & \\
\hline & Kalibaus & Labeo calbasu & & $43.72 \pm 1.67$ & $38.46 \pm 4.23$ & $24.40 \pm 3.42$ & $27.49 \pm 3.68$ & $44.24 \pm 3.65$ & $172.03 \pm 7.32$ & $18.29 \pm 2.89$ & $279.58 \pm 11.21$ & $18.67 \pm 2.57$ & $27.66 \pm 4.23$ & $694.55 \pm 86.71$ & \\
\hline & Bighead carp & $\begin{array}{l}\begin{array}{l}\text { Hypophthalmichthys } \\
\text { nobilis }\end{array} \\
\end{array}$ & & $113.03 \pm 5.11$ & $115.28 \pm 8.24$ & $82.26 \pm 4.63$ & $46.80 \pm 4.35$ & $98.25 \pm 5.68$ & $259.73 \pm 9.78$ & $20.30 \pm 3.65$ & $267.90 \pm 9.43$ & $17.12 \pm 3.21$ & $43.35 \pm 5.43$ & $1064.03 \pm 90.23$ & \\
\hline & Silver carp & $\begin{array}{l}\begin{array}{l}\text { Hypophthalmichthys } \\
\text { molitrix }\end{array} \\
\end{array}$ & & $185.55 \pm 6.23$ & $139.16 \pm 9.25$ & $107.73 \pm 6.34$ & $78.76 \pm 6.54$ & $181.06 \pm 8.43$ & $329.62 \pm 12.32$ & $20.14 \pm 3.56$ & $393.00 \pm 13.24$ & $38.27 \pm 4.53$ & $43.54 \pm 5.23$ & $1516.81 \pm 125.24$ & \\
\hline & $\begin{array}{l}\text { Carfu } \\
\end{array}$ & Cyprinus carpio & & $\begin{array}{l}181.90 \pm 6.12 \\
\end{array}$ & $161.54 \pm 8.34$ & $108.89 \pm 6.32$ & $90.31 \pm 7.34$ & $209.77 \pm 9.98$ & $316.51 \pm 13.24$ & $37.68 \pm 5.22$ & $535.45 \pm 15.46$ & $46.33 \pm 2.45$ & $54.16 \pm 6.34$ & $1742.55 \pm 163.27$ & \\
\hline & Grass carp & Ctenopharyngodon idella & & $123.35 \pm 4.89$ & $72.20 \pm 4.57$ & $49.12 \pm 5.21$ & $52.38 \pm 5.64$ & $39.12 \pm 4.35$ & $176.57 \pm 7.57$ & $17.12 \pm 2.45$ & $360.06 \pm 15.34$ & $23.23 \pm 3.56$ & $18.92 \pm 3.45$ & $932.07 \pm 106.59$ & \\
\hline & Ghonia & Labeo gonius & & $128.00 \pm 6.34$ & $42.70 \pm 4.32$ & $36.40 \pm 5.21$ & $44.53 \pm 4.65$ & $49.10 \pm .34$ & $293.92 \pm 9.45$ & $41.04 \pm 3.87$ & $412.79 \pm 7.67$ & $25.63 \pm 4.67$ & $39.74 \pm 4.57$ & $1113.86 \pm 137.86$ & \\
\hline \multirow{3}{*}{$\begin{array}{l}\text { Barbs and } \\
\text { minnows }\end{array}$} & Chela & Chela cachius & & $37.24 \pm 1.12$ & $2.58 \pm 1.76$ & $1.52 \pm .87$ & 0.00 & $0.50 \pm 112$ & $51.42 \pm 7.56$ & $28.48 \pm 3.56$ & $242.71 \pm 6.54$ & $12.38 \pm 3.21$ & $17.31 \pm 2.54$ & $394.14 \pm 73.54$ & \\
\hline & Sharpunti & Puntius sarana & & $65.13 \pm 1.97$ & $33.76 \pm 1.12$ & $20.25 \pm 1.12$ & $23.84 \pm 3.23$ & $34.36 \pm 3.23$ & $194.33 \pm 8.65$ & $7.57 \pm 1.45$ & $222.97 \pm 9.43$ & $12.83 \pm 3.54$ & $17.69 \pm 3.29$ & $632.74 \pm 78.54$ & \\
\hline & Punti & Puntius sophore & & $178.85 \pm 7.23$ & $19.35 \pm 2.46$ & $22.23 \pm 3.54$ & $54.44 \pm 6.34$ & $20.93 \pm 4.21$ & $419.24 \pm 18.24$ & $32.02 \pm 4.67$ & $784.58 \pm 16.56$ & $50.25 \pm 5.32$ & $48.09 \pm 4.65$ & $1629.98 \pm 251.17$ & \\
\hline \multirow{5}{*}{$\frac{\text { Carplet }}{\text { Eel }}$} & Mola & Amblypharyngodon mola & & $26.08 \pm 3.24$ & $3.12 \pm .80$ & $12.07 \pm 2.32$ & $6.95 \pm 1.21$ & $9.04 \pm 2.34$ & $41.92 \pm 4.65$ & $5.06 \pm 1.54$ & $42.31 \pm 3.65$ & $4.49 \pm 1.21$ & $2.44 \pm .98$ & $153.48 \pm 15.67$ & \\
\hline & Baim & Mastacembelus armatus & Mastacembelidae & $34.03 \pm 1.86$ & $5.11 \pm 1.47$ & $6.68 \pm .97$ & $12.48 \pm 1.65$ & $1.63 \pm .78$ & $87.19 \pm 3.78$ & $11.77 \pm 2.4$ & $303.65 \pm 8.56$ & $16.59 \pm 3.76$ & $17.89 \pm 3.54$ & $497.02 \pm 92.63$ & \multirow{4}{*}{$1819.67 \pm 304.21$} \\
\hline & Tara Baim & Macrognathus aculeatus & & $30.25 \pm .89$ & $3.96 \pm-12$ & $4.32 \pm 1.43$ & $8.65 \pm 1.23$ & $0.83 \pm .32$ & $106.72 \pm 6.45$ & $17.16 \pm 3.89$ & $224.55 \pm 6.56$ & $14.56 \pm 3.45$ & $10.50 \pm 2.69$ & $421.50 \pm 71.25$ & \\
\hline & Guchi baim & Macrognathus pancalus & & $16.40 \pm 1.10$ & $7.23 \pm 2.32$ & $5.14 \pm 1.23$ & $11.62 \pm 2.14$ & $0.37 \pm .12$ & $35.65 \pm 3.68$ & $3.78 \pm 1.21$ & $145.04 \pm 5.45$ & $2.54 \pm .96$ & $6.32 \pm 2.42$ & $234.08 \pm 43.93$ & \\
\hline & Chikra baim & Macrognathus pancalus & & $73.48 \pm 5.67$ & $13.66 \pm 3.56$ & $15.45 \pm 2.65$ & $26.47 \pm 3.65$ & $15.40 \pm 3.21$ & $115.58 \pm 7.45$ & $27.19 \pm 4.56$ & $329.15 \pm 7.85$ & $27.84 \pm 4.53$ & $23.14 \pm 3.62$ & $667.36 \pm 97.74$ & \\
\hline \multirow[t]{14}{*}{ Catfish } & \begin{tabular}{|l|} 
Ayr \\
\end{tabular} & Sperata aor & Bagridae & $38.35 \pm . .98$ & $7.85 \pm 1.24$ & $8.92 \pm .79$ & $16.23 \pm 3.24$ & $4.27 \pm 1.21$ & $87.91 \pm 8.57$ & $15.38 \pm 2.87$ & $372.35 \pm 11.34$ & $22.49 \pm 4.32$ & $19.26 \pm 4.21$ & $593.01 \pm 112.65$ & \multirow{3}{*}{$665.91 \pm 128.60$} \\
\hline & Baga ayr & Bagarius bagarius & & $7.30 \pm .54$ & 0.00 & 0.00 & 0.00 & 0.00 & $14.73 \pm 2.67$ & 0.00 & $50.77 \pm 4.76$ & 0.00 & $0.11 \pm .08$ & $72.90 \pm 16.04$ & \\
\hline & Boal & Wallago attu & Siluridae & \begin{tabular}{|l|l}
$175.00 \pm 5.67$ \\
\end{tabular} & $67.09 \pm 4.76$ & $54.86 \pm 4.94$ & $92.61 \pm 5.65$ & $48.66 \pm 4.67$ & $455.70 \pm 12.34$ & $54.23 \pm 4.34$ & $603.13 \pm 14.34$ & $29.86 \pm 4.52$ & $57.31 \pm 6.47$ & $1638.45 \pm 199.77$ & \\
\hline & Pabda & Ompok pabda & & $10.85 \pm .87$ & $11.91 \pm 1.89$ & $6.42 \pm 1.32$ & $1.47 \pm .98$ & $10.03 \pm 2.34$ & $31.25 \pm 5.65$ & $1.37 \pm .98$ & $49.85 \pm 5.34$ & $3.93 \pm 1.21$ & $3.16 \pm 1.28$ & $130.22 \pm 15.62$ & \multirow{2}{*}{$\begin{array}{l}1768.69 \pm 214.84 \\
215.64 \pm 16.70\end{array}$} \\
\hline & Shing & Heteropneustes fossilis & Heteropneustidae & $27.18 \pm 2.14$ & $33.95 \pm 3.54$ & $21.84 \pm 1.11$ & $4.90 \pm 1.21$ & $29.82 \pm 4.54$ & $36.27 \pm 4.56$ & $4.36 \pm 1.11$ & $50.18 \pm 2.87$ & $2.65 \pm .97$ & $4.49 \pm 1.23$ & $215.64 \pm 16.70$ & \\
\hline & Pangus & \begin{tabular}{|l|}
$\begin{array}{l}\text { Pangasianodon } \\
\text { hypophthalmus }\end{array}$ \\
\end{tabular} & \begin{tabular}{|l|} 
Pangasiidae \\
\end{tabular} & $25.85 \pm 1.13$ & $75.34 \pm 6.98$ & $89.35 \pm 5.32$ & $25.97 \pm 2.34$ & $36.85 \pm 3.75$ & 0.00 & $16.01 \pm 1.78$ & $116.79 \pm 8.56$ & $16.44 \pm 3.42$ & $11.38 \pm 3.45$ & $413.99 \pm 38.76$ & $413.99 \pm 38.76$ \\
\hline & Magur & Clarias batrachus & Clariidae & $18.28 \pm 1.54$ & $20.94 \pm .97$ & $4.97 \pm 1.15$ & $7.28 \pm 2.34$ & $3.31 \pm 1.21$ & $17.30 \pm 4.85$ & $2.06 \pm .87$ & $34.37 \pm 3.23$ & $2.89 \pm .98$ & $2.17 \pm 1.28$ & $113.58 \pm 10.90$ & $113.58 \pm 10.90$ \\
\hline & Shilong & Silonia silondia & Schilbeidae & $3.05 \pm 1.12$ & 0.00 & & & & $8.82 \pm 2.45$ & & $8.29 \pm 2.34$ & $0.07 \pm .01$ & $0.74 \pm .21$ & $20.97 \pm 3.54$ & \\
\hline & Tangra & Mystus armatus & & $111.40 \pm 4.25$ & $12.57 \pm 3.46$ & $11.51 \pm .11$ & $40.16 \pm 5.43$ & $10.94 \pm 3.23$ & $242.09 \pm 15.74$ & $24.53 \pm 2.65$ & $353.58 \pm 9.45$ & $33.77 \pm 3.34$ & $32.15 \pm 6.34$ & $872.69 \pm 117.70$ & \\
\hline & Gulsha & Mystus cavasius & & $55.10 \pm 3.54$ & $9.62 \pm 3.49$ & $8.02 \pm 1.12$ & $27.08 \pm 2.43$ & $13.01 \pm 1.98$ & \begin{tabular}{|l|}
$114.07 \pm 7.86$ \\
\end{tabular} & $15.24 \pm 4.32$ & $351.39 \pm 8.45$ & $19.49 \pm 3.78$ & $17.89 \pm 3.21$ & $630.91 \pm 106.31$ & $117.68 \pm 104.31$ \\
\hline & Batasi & \begin{tabular}{|l|} 
Pseudeutropius \\
atherinoides
\end{tabular} & & $29.50 \pm 1.76$ & $1.60 \pm .97$ & $1.32 \pm .96$ & $2.40 \pm 1.11$ & $0.44 \pm .13$ & $163.83 \pm 6.45$ & $22.73 \pm 3.56$ & $256.63 \pm 7.84$ & $6.77 \pm 2.31$ & $13.27 \pm 4.32$ & $498.49 \pm 87.86$ & $117.68 \pm 104.31$ \\
\hline & Bacha & \begin{tabular}{|l} 
Eutroropiches \\
\end{tabular} & & $7.90 \pm 2.46$ & 0.00 & $0.69 \pm .21$ & 0.00 & 0.00 & $16.88 \pm 4.65$ & $2.19 \pm .98$ & $63.03 \pm 3.56$ & $1.22 \pm .63$ & $2.70 \pm 1.02$ & $94.61 \pm 19.55$ & \\
\hline & Gharua & \begin{tabular}{|l} 
Clupisoma garua \\
\end{tabular} & Ailiidae & $15.20 \pm 3.25$ & 0.00 & 0.00 & 0.00 & 0.00 & $25.95 \pm 3.45$ & $11.20 \pm 2.56$ & $103.06 \pm 4.56$ & $10.27 \pm 2.43$ & $12.29 \pm 2.41$ & $177.97 \pm 31.16$ & \\
\hline & kajuli & Ailia coila & & $8.58 \pm .35$ & $0.18 \pm .18$ & $0.18 \pm .09$ & $0.26 \pm .07$ & $0.79 \pm .14$ & $29.53 \pm 4.65$ & $6.25 \pm 1.76$ & $87.77 \pm 5.63$ & $10.54 \pm 1.64$ & $1.91 \pm .98$ & $145.99 \pm 27.23$ & $23.96 \pm 58.22$ \\
\hline \multirow{3}{*}{ Snakeheads } & Shol & Channa striata & Channidae & $23.23 \pm 1.23$ & $12.14 \pm .99$ & $5.89 \pm .98$ & $4.29 \pm 1.12$ & $5.11 \pm 1.89$ & $45.74 \pm 4.32$ & $7.66 \pm 2.43$ & $122.38 \pm 5.43$ & $6.31 \pm 1.42$ & $6.37 \pm 2.51$ & $239.11 \pm 36.90$ & \multirow{3}{*}{$950.3 \pm 131.34$} \\
\hline & Gozar & Channa marulius & & $10.30 \pm .76$ & $0.69 \pm .54$ & $0.09 \pm .02$ & $2.02 \pm .98$ & 0.00 & $25.26 \pm 3.78$ & $2.42 \pm 1.23$ & $81.91 \pm 4.54$ & $2.65 \pm 1.87$ & $3.91 \pm 1.11$ & $129.24 \pm 25.42$ & \\
\hline & Taki & Channa punctata & & $91.55 \pm 3.45$ & $18.34 \pm 2.45$ & $11.16 \pm 1.14$ & $25.10 \pm 32$ & $2.64 \pm 1.21$ & $184.30 \pm 14.6$ & $14.95 \pm 3.56$ & $195.79 \pm 4.65$ & $13.22 \pm 3.76$ & $24.88 \pm 4.23$ & $581.93 \pm 73.74$ & \\
\hline \multirow[t]{4}{*}{ Perches } & Tilapia & Oreochromis niloticus & Cichlidae & $48.03 \pm 2.12$ & $80.64 \pm 5.34$ & $64.40 \pm 3.54$ & $40.10 \pm 4.32$ & $115.39 \pm 5.78$ & $89.17 \pm 3.86$ & $18.31 \pm 2.13$ & $157.67 \pm 7.65$ & $20.39 \pm 2.54$ & $14.38 \pm 4.65$ & $648.47 \pm 46.66$ & \multirow{5}{*}{\begin{tabular}{|l|}
$648.47 \pm 46.66$ \\
$144.63 \pm 12.69$ \\
$72.75 \pm 9.46$ \\
$1156.30 \pm 195.50$ \\
\end{tabular}} \\
\hline & Koi & Anabas testudineus & Anabantidae & $21.36 \pm 2.63$ & $7.81 \pm 2.32$ & $8.36 \pm .89$ & $10.41 \pm 1.67$ & $7.89 \pm 2.32$ & $31.11 \pm 2.46$ & $9.31 \pm 1.76$ & $41.26 \pm 4.65$ & $2.54 \pm .79$ & $4.58 \pm 1.34$ & $144.63 \pm 12.69$ & \\
\hline & Khalisha & Colisa fasciata & Osphronemidae & $11.35 \pm .80$ & $0.78 \pm .23$ & $1.79 \pm .02$ & $3.78 \pm 1.56$ & $3.00 \pm 1.21$ & $16.78 \pm 2.34$ & $2.60 \pm . .64$ & $29.78 \pm 2.54$ & $1.66 \pm .89$ & $1.25 \pm .65$ & \begin{tabular}{|l|l|}
$72.78 \pm 9.46$ \\
\end{tabular} & \\
\hline & Chanda & Chanda nama & Ambassidae & $104.40 \pm 5.75$ & $10.60 \pm 2.39$ & $10.22 \pm 1.16$ & $40.97 \pm 3.62$ & $5.73 \pm 1.13$ & $281.6 \pm 10.23$ & $28.92 \pm 3.58$ & $618.94 \pm 11.32$ & $20.19 \pm 3.78$ & $34.71 \pm 5.43$ & $1156.30 \pm 195.50$ & \\
\hline \multirow{2}{*}{ Featherbacks } & Chital & Chitala chitala & Notopteridae & 0.00 & 0.00 & 0.00 & 0.00 & 0.00 & 0.00 & 0.00 & $11.67 \pm 2.34$ & $0.14 \pm .032$ & $0.22 \pm .12$ & $12.02 \pm 3.68$ & \\
\hline & Foli & Notopterus notopterus & Notopteridae & $7.351 \pm .76$ & 0.00 & 0.00 & 0.00 & 0.00 & $8.93 \pm 3.45$ & 0.00 & $24.63 \pm 3.54$ & 0.00 & $0.67 \pm .12$ & $41.58 \pm 7.94$ & \multirow{4}{*}{\begin{tabular}{|l|}
$53.61 \pm 11.38$ \\
$262.97 \pm 55.32$ \\
$713.17 \pm 130.14$ \\
\end{tabular}} \\
\hline Needlefish & Kakila & Xenentodon cancila & Belonidae & $17.75 \pm 1.11$ & $4.11 \pm 1.28$ & $4.55 \pm 1.25$ & $13.80 \pm 1.78$ & $0.15 \pm .06$ & $26.01 \pm 3.65$ & $3.85 \pm 1.24$ & $182.13 \pm 6.45$ & $6.07 \pm 1.54$ & $4.56 \pm 1.23$ & $262.97 \pm 55.32$ & \\
\hline \begin{tabular}{|l|l|} 
Goby \\
\end{tabular} & Balia & 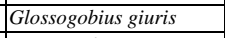 & Gobiidae & $68.48 \pm 3.14$ & $8.18 \pm 1.76$ & $6.7 \pm 1.13$ & $30.96 \pm 3.76$ & $5.82 \pm 2.75$ & $68.5 \pm 5.63$ & $35.64 \pm 3.45$ & $435.95 \pm 8.76$ & $20.33 \pm 2.95$ & $32.61 \pm 5.79$ & $713.17 \pm 130.14$ & \\
\hline & Kachki & Corica soborna & Clupeidae & & & $19.34 \pm 1.46$ & & & & & & 55.43 & & $1085.99 \pm 150.53$ & \\
\hline \multirow[t]{2}{*}{ Shad } & Chapila & Gudusia chapra & & $10.75 \pm 1.16$ & $5.57 \pm 1.56$ & $6.15 \pm 1.21$ & $17.66 \pm 2.13$ & $6.42 \pm 1.21$ & $71.15 \pm 4.65$ & $13.69 \pm 4.54$ & $309.20 \pm 7.54$ & $9.53 \pm 3.64$ & $13.79 \pm 2.43$ & $463.92 \pm 94.37$ & \multirow{2}{*}{$1588.61 \pm 248.92$} \\
\hline & Ilish & Tenualosa ilisha & & $2.98 \pm .15$ & $0.07 \pm .01$ & $0.07 \pm .01$ & $0.15 \pm .09$ & 0.00 & $8.09 \pm 1.11$ & $1.57 \pm .56$ & $25.10 \pm 4.32$ & $0.28 \pm .19$ & $0.39 \pm .12$ & $38.70 \pm 7.87$ & \\
\hline loach & Rani mach & Botia dario & Botiidae & 0.00 & $0.25 \pm .14$ & $0.25 \pm .13$ & $1.21 \pm$ & 0.00 & $0.83=$ & 0.00 & $5.12 \pm 1.34$ & $0.32 \pm .12$ & $0.25 \pm .12$ & $8.23 \pm 1.56$ & $8.23 \pm 1.56$ \\
\hline Leaffish & \begin{tabular}{|l|l|} 
Veda \\
\end{tabular} & Nandus nandus & \begin{tabular}{|l|} 
Nandidae \\
\end{tabular} & $4.06 \pm, 98$ & $0.25 \pm .12$ & $1.33 \pm .42$ & $0.81 \pm .18$ & 0.00 & $6.00 \pm 1.21$ & 0.00 & $83.92 \pm 2.67$ & $1.81 \pm .97$ & $2.04 \pm 1.11$ & $100.20 \pm 26.03$ & $100.20 \pm 26.03$ \\
\hline Prawn & Golda chingri & \begin{tabular}{|l|}
$\begin{array}{l}\text { Macrobrachium } \\
\text { rosenbergii }\end{array}$ \\
\end{tabular} & Palaemonidae & $12.84 \pm 1.19$ & $0.38 \pm .14$ & $0.38 \pm .12$ & $4.31 \pm 1.23$ & $1.61 \pm .97$ & $107.27 \pm 9.46$ & $6.89 \pm 2.12$ & $132.19 \pm 6.76$ & $6.72 \pm 1.36$ & $2.48 \pm 1.21$ & $275.05 \pm 49.10$ & \\
\hline & Choto chingri & \begin{tabular}{|l|} 
Macrobrachium \\
malcomsonii
\end{tabular} & & $74.28 \pm 2.43$ & $5.50 \pm 1.23$ & $3.12 \pm .08$ & $24.27 \pm .32$ & $1.06 \pm .89$ & $217.48 \pm 9.45$ & $35.02 \pm 4.23$ & $353.23 \pm 7.57$ & $20.08 \pm 5.12$ & $36.79 \pm 6.32$ & $770.80 \pm 116.32$ & \\
\hline
\end{tabular}

$\mathrm{Tl}=$ Tarail, $\mathrm{Kd}=$ Kotiadi, $\mathrm{Krg}=$ Karimganj, $\mathrm{Kg}=$ kargaon, $\mathrm{Dd}=$ Dhuldia, $\mathrm{Cg}=$ Chouganga, $\mathrm{Mk}=$ morich khali, Chg = chamra ghat, $\mathrm{Nl}=\mathrm{Nikli}, \mathrm{Rp}=\mathrm{Rodar}$ podda, $\mathrm{spp} .=$ species. 
Table 3. Trends in fish catch by the fishermen involved in FLCs of Kishoreganj, 2018.

\begin{tabular}{|c|c|c|c|c|c|c|c|c|}
\hline SI & FLCs & Upazila & $\begin{array}{l}\text { Source } \\
\text { of catch }\end{array}$ & $\begin{array}{l}\text { Catch/ day } \\
\text { (kg) }\end{array}$ & \begin{tabular}{|l} 
Catch \\
duration \\
$(\mathrm{mon} / \mathrm{y})$
\end{tabular} & \begin{tabular}{|l|} 
Catch \\
Duration \\
(day/mon)
\end{tabular} & $\begin{array}{l}\text { No. of } \\
\text { Fishers }\end{array}$ & $\begin{array}{l}\text { Total fish catch } \\
\text { (MT) }\end{array}$ \\
\hline 1 & Chamraghat & Karimganj & Haor & 25.7 & 10 & 27 & 535 & 3513.68 \\
\hline 2 & Morichkhali & Karimganj & Haor & 7.33 & 7 & 28 & 250 & 340.89 \\
\hline 3 & Mach Mohal & Karimganj & Haor & 30.94 & 11 & 23 & 70 & 545.33 \\
\hline 4 & Dhuldia & Kotiadi & Haor & 5 & 12 & 24 & 80 & 112.80 \\
\hline 5 & Korgaon & Kotiadi & Haor & 39.64 & 8 & 26 & 175 & 1441.09 \\
\hline 6 & Kotiadi Bazar & Kotiadi & Haor & 21.58 & 12 & 28 & 220 & 1576.39 \\
\hline 7 & RodarPodda & Nikli & Haor & 17.17 & 8 & 26 & 250 & 904.17 \\
\hline 8 & Nutun Bazar & Nikli & Haor & 26 & 9 & 25 & 160 & 907.88 \\
\hline 9 & Chowganga & Itna & Haor & 12.5 & 7 & 27 & 350 & 856.41 \\
\hline \multirow[t]{2}{*}{10} & Tarail Mach Bazar & Taril & Haor & 21.67 & 11 & 30 & 345 & 2375.57 \\
\hline & \multicolumn{2}{|l|}{ Total } & & $207.53 \pm 11.30$ & 93 & 263.19 & 2435 & $12574.21 \pm 1029.64$ \\
\hline
\end{tabular}

Table 4. Fish landing in different FLCs of Kishoreganj haor as perceived by the Aratdars.

\begin{tabular}{|c|c|c|c|c|c|c|c|}
\hline \multirow[t]{2}{*}{ SI } & \multirow[t]{2}{*}{ Name of FLCs } & \multirow[t]{2}{*}{ Upazila } & \multicolumn{2}{|c|}{ Seasonal landing/day (Kg) } & \multirow{2}{*}{$\begin{array}{l}\text { Average } \\
\text { landing/ day } \\
(\mathrm{Kg})\end{array}$} & \multirow{2}{*}{$\begin{array}{l}\text { Av. landing/ } \\
\text { month (Kg) }\end{array}$} & \multirow{2}{*}{$\begin{array}{l}\text { Total landing } \\
\text { /year }(\mathrm{Kg})\end{array}$} \\
\hline & & & $\begin{array}{l}\text { Peak season } \\
\text { (Jun-Oct) }\end{array}$ & \begin{tabular}{|l|} 
Lean season \\
(Nov-Jan)
\end{tabular} & & & \\
\hline 1 & Dhuldia & Kotiadi & 4400 & 2684.64 & 3542.32 & 106269.6 & 850156.8 \\
\hline 2 & Chamraghat & Karimganj & 26977.78 & 22163.24 & 24570.51 & 737115.3 & 5896922 \\
\hline 3 & Mach Mohal & Karimganj & 2630 & 1234.37 & 1932.18 & 57965.55 & 463724.4 \\
\hline 4 & Morichkhali & Karimganj & 944.44 & 458.72 & 701.58 & 21047.4 & 168379.2 \\
\hline 5 & Taril Mach Bazar & Taril & 11464.29 & 6356.23 & 8910.26 & 267307.8 & 2138462 \\
\hline 6 & Korgaon & Kotiadi & 4266.66 & 3457.36 & 3862.01 & 115860.3 & 926882.4 \\
\hline 7 & Rodar Podda & Nikli & 2914 & 2176.43 & 2545.21 & 76356.45 & 610851.6 \\
\hline 8 & Kotiadi Bazar & Kotiadi & 9040 & 8754.23 & 8897.11 & 266913.5 & 2135308 \\
\hline 9 & Chowganga & Itna & 9250 & 5485.12 & 7367.56 & 221026.8 & 1768214 \\
\hline \multirow[t]{2}{*}{10} & Nutun Bazar & Nikli & 5500 & 1468.83 & 3484.41 & 104532.5 & 836259.6 \\
\hline & \multicolumn{2}{|l|}{ Total (Kg) } & $\begin{array}{l}\text { 77387.17 } \pm 754 \\
0.64\end{array}$ & $\begin{array}{l}54239.17 \pm 642 \\
8.53\end{array}$ & \begin{tabular}{|l|}
$65813.17 \pm 6941$ \\
.92
\end{tabular} & $\begin{array}{l}1974395 \pm 20825 \\
7.75\end{array}$ & $\begin{array}{l}15795161 \pm 16660 \\
61.93\end{array}$ \\
\hline
\end{tabular}

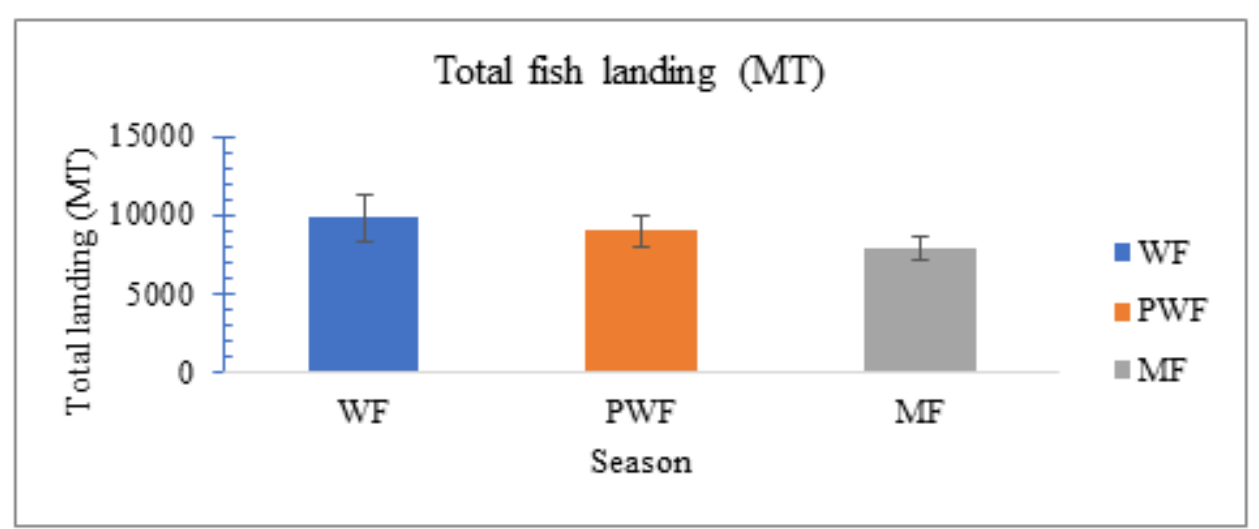

$\mathrm{WF}=$ Winter fishing, $\mathrm{PWF}=$ Pre-winter fishing, $\mathrm{MF}=$ Monsoon fishing, $\mathrm{MT}=$ Metric ton

Figure 5. Landed fish in different fishing season.

\subsection{Status of harvested fish}

The harvested fish in Kishoreganj haor is sold by fisherman to local arat, local paiker and the retail market. In total, about $88.35 \%$ fisherman sell their captured fish at local arat and rest of the fishers $(11.65 \%)$ sell fish to paiker, retail market and consumption. About $100 \%$ fishermen sell the captured fish to local arat in Dhuldiya, Tarial, Rodar podda, Kotiyadi and Nikli. Only $13.55 \%$ fisherman sell the harvested fish to local paiker and a large portion of fisherman $(86.45 \%)$ give the fish to seasonal paiker. Only $14.78 \%$ fisherman take their captured fish in local fish market for selling but $85.22 \%$ fisherman give their fish to paiker who come from different 
places (Table 5). Hossain et al., 2013, stated that fisherman catches fish independently and sells to the Aratder. Paiker purchases from the Aratder (98\%) and fisherman (2\%). Fisherman sells $80.5 \%$ of the fish to the Aratder and consumer $(10 \%)$. Aratder sells fish to the secondary Aratder (75\%), paiker (10\%), processor (5\%), wholesaler (8\%) and retailer (2\%). Paiker sells to wholesaler (95\%) and retailer (5\%). These findings are mostly complying with present findings. In Kishoreganj haor, 56.6\% fisherman clean the captured fish by water and 43.4\% fisherman do not use water for washing fish before selling (Table 5). Nowsad, 2010 reported that only giant prawn, penaeid shrimp and some other marine high valued species were found to be washed adequately (75-100\%), while washing of most of the freshwater species before marketing were in $20-40 \%$ range. After harvesting, $45.62 \%$ fishers use fresh utensil for carrying fish to arat and $54.38 \%$ use contaminated utensil. $100 \%$ fishers use fresh container and contaminated container in Dhuldiya and Rodar podda respectively (Table 5). Table 6 shows that everyday a fisherman harvest in an average $23.9 \mathrm{~kg}$ of fish in Kishoreganj haor area. Among these, fisherman sales in an average $22.26 \mathrm{~kg}$ of fish per day at arat, paiker and retail market and keeps only 1.64 $\mathrm{kg}$ for their daily consumption. Among the landing centers, the fisherman harvest more than $30 \mathrm{~kg}$ of fish per day in karimganj, kargaon and Nikli area as well as less than $10 \mathrm{~kg}$ of fish is collected in Dhuldia (5 $\mathrm{kg}$ ) and Morichkhali $(7.3 \mathrm{~kg})$. The consumption rate of fish by fisherman household is comparatively high in Korgaon $(3.3 \mathrm{~kg})$ and Nikli $(2.6 \mathrm{~kg})$ and low in Dhuldiya $(.75 \mathrm{~kg})$ and Morichkhali $(0.6 \mathrm{~kg})$. There is no such types of recorded data, that why it's not possible to compare.

Table 5. Status of harvested fish (\%).

\begin{tabular}{|c|c|c|c|c|c|c|c|c|c|c|}
\hline \multirow{3}{*}{ FLC } & \multicolumn{6}{|c|}{ Selling to } & \multirow{2}{*}{\multicolumn{2}{|c|}{$\begin{array}{c}\text { Washing by } \\
\text { water }\end{array}$}} & \multirow{2}{*}{\multicolumn{2}{|c|}{$\begin{array}{c}\text { Transporting with } \\
\text { fresh utensil } \\
\end{array}$}} \\
\hline & \multicolumn{2}{|c|}{ LA } & \multicolumn{2}{|c|}{ LP } & \multicolumn{2}{|c|}{ RM } & & & & \\
\hline & Yes & No & Yes & No & Yes & No & Yes & No & Yes & No \\
\hline Dhuldiya & 100 & 0 & 50 & 50 & 0 & 100 & 100 & 0 & 100 & 0 \\
\hline Chamta ghat & 60 & 40 & 10 & 90 & 10 & 90 & 40 & 60 & 33.33 & 66.67 \\
\hline Karimgonj & 88.89 & 11.11 & 22.22 & 77.78 & 55.56 & 44.44 & 66.67 & 33.33 & 55.56 & 44.44 \\
\hline Morichkhali & 88.89 & 11.11 & 0 & 100 & 0 & 100 & 55.56 & 44.44 & 44.44 & 55.56 \\
\hline Tarial & 100 & 0 & 33.33 & 66.67 & 22.22 & 77.78 & 33.33 & 66.67 & 33.33 & 66.67 \\
\hline Kargaon & 85.71 & 14.29 & 0 & 100 & 0 & 100 & 57.14 & 42.86 & 42.86 & 57.14 \\
\hline Rodar poddar & 100 & 0 & 0 & 100 & 0 & 100 & 66.67 & 33.33 & 0 & 100 \\
\hline Kotiyadi & 100 & 0 & 0 & 100 & 0 & 100 & 66.67 & 33.33 & 66.67 & 33.33 \\
\hline Chouganga & 100 & 0 & 20 & 80 & 40 & 60 & 40 & 60 & 40 & 60 \\
\hline Nikli & 60 & 40 & 0 & 100 & 20 & 80 & 40 & 60 & 40 & 60 \\
\hline In total & 88.35 & 11.65 & 13.55 & 86.45 & 14.78 & 85.22 & 56.6 & 43.4 & 45.62 & 54.38 \\
\hline
\end{tabular}

LA = Local arat, LP = Local paiker, RM = Retail market

Table 6. Status of harvested, selling and consumed fish per fisherman.

\begin{tabular}{|l|l|l|l|}
\hline FLC & Quantity of Harvest (Kg/Day) & Quantity of Sale (Kg/Day) & Quantity of Consume (Kg/Day) \\
\hline Dhuldia & $5 \pm 0$ & $4.25 \pm 0.4$ & $0.75 \pm 0.4$ \\
\hline Chamta & $23.7 \pm 27.1$ & $22.4 \pm 26.2$ & $1.4 \pm 1.4$ \\
\hline Karimganj & $39.5 \pm 30.7$ & $37.8 \pm 29.5$ & $1.7 \pm 1.7$ \\
\hline Morichkhali & $7.3 \pm 4.8$ & $6.7 \pm 4.7$ & $0.6 \pm 0.3$ \\
\hline Tarial & $21.6 \pm 18.4$ & $19.9 \pm 17.7$ & $1.7 \pm 1.1$ \\
\hline Korgaon & $41.1 \pm 10.1$ & $37.6 \pm 11.4$ & $3.3 \pm 1.7$ \\
\hline Rodarpudda & $17 \pm 13$ & $15.7 \pm 12.4$ & $1.3 \pm 0.8$ \\
\hline Kotiadi & $22.5 \pm 7.3$ & $20.8 \pm 6.6$ & $1.7 \pm 1.2$ \\
\hline Chowganga & $15.9 \pm 8.4$ & $14.6 \pm 7.3$ & $1.3 \pm 1.72$ \\
\hline Nikli & $45.4 \pm 32.4$ & $42.8 \pm 30.9$ & $2.6 \pm 1.5$ \\
\hline In total & $\mathbf{2 3 . 9} \pm \mathbf{1 3 . 9 6}$ & $\mathbf{2 2 . 2 6} \pm \mathbf{1 3 . 2 3}$ & $\mathbf{1 . 6 4} \pm \mathbf{0 . 8 1}$ \\
\hline
\end{tabular}

\subsection{Fish transportation}

After harvesting, various types of equipment are used for transportation of fish from water body to arat. Table 7 shows that average $45.2 \%$ fish is transported by boat, $21.9 \%$ by bamboo basket, and $14.8 \%$ by plastic crate. A small portion of fish is transported by ice box (4.7\%), aluminium pot (2.7\%) and gunny bag (3.2\%). In Dhuldia $100 \%$ fish is transported by boat. Above 50\% captured fish is carried away by boat in Morichkhali and Chowganga. 54.5\% fish is carried away by bamboo basket in arat after harvesting from haor. During transportation, ice box is used only in Chamta ghat (6.7\%) and Rodarpodda (40\%) along with plastic crate and 
plastic drum. Boat, plastic crate and bamboo basket are available in all fish landing centers after harvesting of fish. Nowsad, 2010 stated that most of the fishes are transported by bamboo baskets of different shapes and sizes, with or without hogla mat or polythene covered and with or without ices. Fish are also being transported by plastic drum, steel made half-drum, country boat partitioned by hogla mat, aluminium container with or without lid, fibre glass crater, plastic crater, styrofoam box and ideal ice boxes. Ilish landed in Barguna-Barishal coast are mainly transported in water-ways by country boat. These are mostly complying with our finding. It is found from Kishoreganj haor basin that 32.6\% fisherman use ice and $67.4 \%$ fisherman do not use ice after harvesting of fish. Only $7 \%$ fisherman use ice in fish immediately after harvesting. About $15.6 \%$ and $6.3 \%$ fisherman use ice within 4 to 8 hours and 1 to 3 hours respectively after harvesting. About $3.7 \%$ fisherman use ice after 8 hours later. After arrival of fish at arat only $29.6 \%$ ice is still remaining and $70.4 \%$ ice is melted before arrival. $100 \%$ ice is melted before reaching of fish at arat in Karimganj, Morichkhali, Kotiadi, Chawganga and Nikli. In Kargaon and Rodar podda $100 \%$ used ice in fish is still present after reaching at arat (Table 8, Figure 6). There is a variation in quantity of fish transportation according to season and off-season. In fishing season about $681.73 \mathrm{~kg}$ fish was daily transported by traders. On the other hand, $370.65 \mathrm{~kg}$ fish was daily transported by traders in off-season in haor. In this study found that, $91.3 \%$ traders use ice for fish preservation in fishing season where $89.1 \%$ traders use in off-season. About $8.7 \%$ and $10.9 \%$ traders did not use any ice in season and off-season respectively. In fishing season about $37.68 \mathrm{~kg}$ ice used in fish preservation per day where $25.2 \mathrm{~kg}$ used in off-season. In this survey found that there is available ice for preservation of fish (Table 9). Table 10 shows that, during fishing season about $3972.3 \mathrm{~kg}$ of fish was transported with $1517.13 \mathrm{~kg}$ of ice which ratio is $2.62: 1$. On the other hand, during off-season about $2092.48 \mathrm{~kg}$ of fish was transported with $959.96 \mathrm{~kg}$ of ice which ratio is $2.18: 1$. Among the fish landing centers, the amount of ice used was lowest in Chamta ghat during fishing season where Morichkhali was highest. On the other hand, during off-season, the amount of ice used was lowest in Karimganj where Morichkhali was highest. Nowsad, 2010 noted that only $12 \%$ fishermen use very minimal quantity of ice in fish (ice-fish ratio of $1: 3$ or $1: 4$ ) and $88 \%$ fishermen and $77 \%$ fish farmer do not use ice in fish at all. All wholesalers/transporters use ice in fish but only $12 \%$ of them use an ice-fish ratio of $1: 1$ and $44 \%$ use a ratio of $1: 2$ but most of wholesalers, retailers and vendors use an ice:fish ratio of 1:3 to 1:5, which is quite negligible. Ice are used in inland fish transport in fish boxes or trucks and on board mechanized boats which carry fish more than a day. Sixty to eighty cans of ice (4.8-6.4 tones) are generally used on 7/8-day trip to ice 5-6 tons of ilish (Nowsad et al., 2012)

Table 7. Fish transportation $(\%)$ in arat.

\begin{tabular}{|c|c|c|c|c|c|c|c|c|}
\hline \multirow[b]{2}{*}{ FLC } & \multicolumn{8}{|c|}{ Equipment used after harvesting } \\
\hline & Boat & Ice Box & $\begin{array}{l}\text { Bamboo } \\
\text { Basket }\end{array}$ & $\begin{array}{l}\text { Steel } \\
\text { Drums }\end{array}$ & $\begin{array}{l}\text { Plastic } \\
\text { Drums }\end{array}$ & $\begin{array}{l}\text { Plastic } \\
\text { Crate }\end{array}$ & $\begin{array}{l}\text { Aluminum } \\
\text { Pot }\end{array}$ & Gunny Bag \\
\hline Dhuldia & 100 & 0 & 0 & 0 & 0 & 0 & 0 & 0 \\
\hline Chamta & 40 & 6.7 & 20 & 0 & 20 & 13.3 & 0 & 0 \\
\hline Karimganj & 30 & 0 & 30 & 0 & 10 & 20 & 0 & 10 \\
\hline Morichkhali & 53.8 & 0 & 7.7 & 0 & 0 & 7.7 & 15.4 & 15.4 \\
\hline Tarial & 35.3 & 0 & 29.3 & 0 & 5.9 & 11.8 & 11.8 & 5.9 \\
\hline Korgaon & 41.7 & 0 & 33.3 & 0 & 0 & 25 & 0 & 0 \\
\hline Rodarpudda & 40 & 40 & 0 & 0 & 0 & 20 & 0 & 0 \\
\hline Kotiadi & 9.1 & 0 & 54.5 & 9.1 & 0 & 27.3 & 0 & 0 \\
\hline Chowganga & 62.5 & 0 & 25 & 0 & 0 & 12.5 & 0 & 0 \\
\hline Nikli & 40 & 0 & 20 & 0 & 30 & 10 & 0 & 0 \\
\hline Average (\%) & 45.2 & 4.7 & 21.9 & 0.9 & 6.6 & 14.8 & 2.7 & 3.2 \\
\hline
\end{tabular}


Table 8. Ice used in fish during transportation (\%).

\begin{tabular}{|c|c|c|c|c|c|c|c|}
\hline \multirow[t]{3}{*}{ FLC } & \multicolumn{5}{|c|}{ Use of ice in fish transportation } & \multicolumn{2}{|c|}{$\begin{array}{l}\text { Remaining ice until } \\
\text { arrival of fish at arat }\end{array}$} \\
\hline & \multirow[b]{2}{*}{ No } & \multicolumn{4}{|c|}{ Yes } & \multirow{2}{*}{ Yes } & \multirow[t]{2}{*}{ No } \\
\hline & & Immediately & Within 1 to $3 \mathrm{hrs}$. & Within 4 to $8 \mathrm{hrs}$. & $>8 \mathrm{hrs}$. & & \\
\hline Dhuldia & 100 & 0 & 0 & 0 & 0 & 0 & 0 \\
\hline Chamta ghat & 72.7 & 9.1 & 9.1 & 9.1 & 0 & 33.3 & 66.7 \\
\hline Karimganj & 77.8 & 0 & 22.2 & 0 & 0 & 0 & 100 \\
\hline Morichkhali & 0 & 0 & 0 & 83.3 & 16.7 & 0 & 100 \\
\hline Tarial & 66.7 & 11.1 & 22.2 & 0 & 0 & 33.3 & 66.7 \\
\hline Korgaon & 83.3 & 16.7 & 0 & 0 & 0 & 100 & 0 \\
\hline Rodar podda & 66.7 & 33.3 & 0 & 0 & 0 & 100 & 0 \\
\hline Kotiadi & 66.7 & 0 & 0 & 33.3 & 0 & 0 & 100 \\
\hline Chowganga & 60 & 0 & 0 & 20 & 20 & 0 & 100 \\
\hline Nikli & 80 & 0 & 10 & 10 & 0 & 0 & 100 \\
\hline In Total & 67.4 & 7 & 6.3 & 15.6 & 3.7 & 29.6 & 70.4 \\
\hline
\end{tabular}

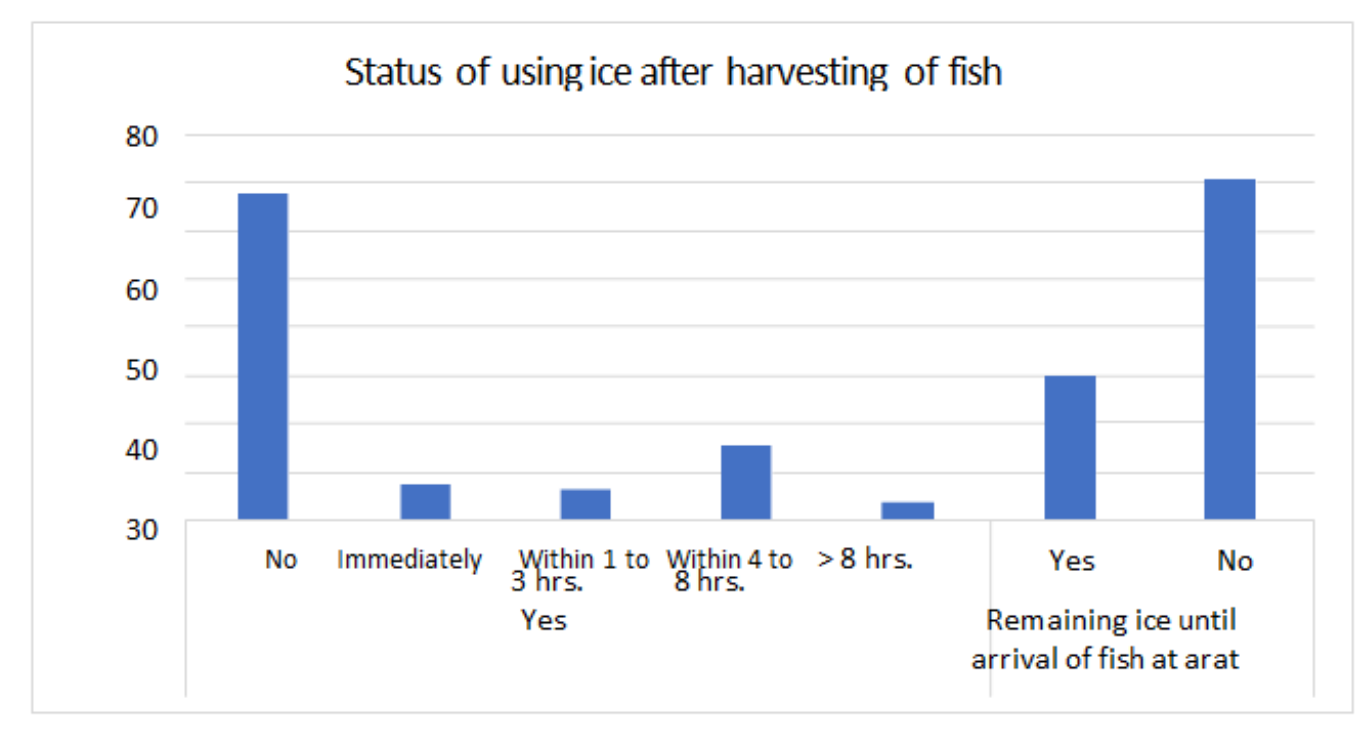

Figure 6. Status of using ice in harvested fish in haor basin.

Table 9. Transportation of fish by fish traders.

\begin{tabular}{|l|l|l|l|l|l|l|}
\hline & Quantity of fish Transportation & \multicolumn{2}{|l|}{ Use of Ice (\%) } & Quantity of Ice Use & \multicolumn{2}{l|}{ Ice Availability (\%) } \\
\cline { 3 - 4 } \cline { 5 - 7 } & (Kg/Day) & Yes & No & (Kg/Day) & Yes & No \\
\hline Season & 681.73 & 91.3 & 8.7 & 37.68 & 100 & 0 \\
\hline Off-Season & 370.65 & 89.1 & 10.9 & 25.2 & 100 & 0 \\
\hline
\end{tabular}

Table 10. Ratio of ice used during transportation of fish in Kishoreganj.

\begin{tabular}{|l|l|l|l|l|l|l|}
\hline \multirow{2}{*}{ FLC } & \multicolumn{3}{|c|}{ Season } & \multicolumn{3}{c|}{ Off-season } \\
\cline { 2 - 7 } & $\begin{array}{l}\text { Transport of } \\
\text { fish (Kg) }\end{array}$ & $\begin{array}{l}\text { Amount of ice } \\
\text { used }\end{array}$ & Ratio & $\begin{array}{l}\text { Transport of fish } \\
(\mathbf{K g})\end{array}$ & $\begin{array}{l}\text { Amount of ice } \\
\text { used }\end{array}$ & Ratio \\
\hline Dhuldiya & 80.71 & 38.29 & $2.11: 1$ & 69.29 & 34.86 & $1.99: 1$ \\
\hline Chamta ghat & 1090 & 202 & $5.39: 1$ & 231.67 & 153.67 & $1.51: 1$ \\
\hline Karimgonj & 267.95 & 52 & $5.15: 1$ & 235 & 46.91 & $5.00: 1$ \\
\hline Morichkhali & 221.43 & 165.43 & $1.34: 1$ & 232.14 & 179.71 & $1.29: 1$ \\
\hline Tarial & 594.29 & 257.71 & $2.31: 1$ & 223.71 & 89.14 & $2.51: 1$ \\
\hline Kargaon & 349.17 & 128 & $2.73: 1$ & 173.33 & 72 & $2.41: 1$ \\
\hline Rodar poddar & 131.67 & 67.83 & $1.94: 1$ & 86.17 & 51 & $1.69: 1$ \\
\hline Kotiyadi & 140 & 62.2 & $2.25: 1$ & 97 & 36 & $2.69: 1$ \\
\hline Chouganga & 163.75 & 37 & $4.43: 1$ & 77.5 & 30 & $2.58: 1$ \\
\hline
\end{tabular}




\begin{tabular}{|l|l|l|l|l|l|l|}
\hline Nikli & 933.33 & 506.67 & $1.84: 1$ & 666.67 & 266.67 & $2.50: 1$ \\
\hline In total & $\mathbf{3 9 7 2 . 3}$ & $\mathbf{1 5 1 7 . 1 3}$ & $\mathbf{2 . 6 2 : 1}$ & $\mathbf{2 0 9 2 . 4 8}$ & $\mathbf{9 5 9 . 9 6}$ & $\mathbf{2 . 1 8 : 1}$ \\
\hline
\end{tabular}

\subsection{Status of arat}

In Kishoreganj haor basin, average 18.7 arat with an area of $41.11 \pm 9.77$ decimal is found in each fish landing center. The highest number of arat is present in Chamta ghat with an area of $55 \pm 1.44$ decimal and lowest in Nikli with an area of $25 \pm 8.42$ decimal (Table 11). In fishing season, everyday about $88 \pm 25$ and $410 \pm 97$ number of fishermen selling captured fish in each arat and fish landing centers respectively (Table 11). The number of fishermen selling fish in arat per day is higher (226) in Morichkhali and Kargaon and lower (8) in Tarail and Rodar podda (Table 11). In fish landing centers, more than 1000 fisherman selling fish in Chamta ghat per day. In total, $92 \%$ fishermen selling fish at arat and only $8 \%$ at paiker (Table 11, Figure 7). Among the fish land centers, more than $90 \%$ fishermen selling their fish in arat except Karimganj (81\%), Tarail (85\%) and Chouganga (83.75\%). The highest 19\% fishermen selling their harvested fish in paiker at Karimganj and lowest in Nikli (0\%) (Table 11). It is seen from the findings that average $617 \mathrm{~kg}$ and $7739 \mathrm{~kg}$ fish is sold by fishermen in each arat and fish landing center every day in fishing season respectively. The highest $1013 \mathrm{~kg}$ fish are sold at arat per day in Nikli and lowest $330 \mathrm{~kg}$ in Chouganga. The highest $26978 \mathrm{~kg}$ fish are sold at fish landing center per dat by fishermen in Chamta ghat and lowest $2630 \mathrm{~kg}$ in Karimganj. There is no published record or study report that showed the status of arat in Kishoreganj haor area. Therefore it was not possible to compare the present findings with any dependable previous sources.

Table 11. Status of arat.

\begin{tabular}{|c|c|c|c|c|c|c|c|c|}
\hline \multirow[t]{2}{*}{$\begin{array}{l}\text { Name of } \\
\text { FLC }\end{array}$} & \multirow{2}{*}{$\begin{array}{l}\text { No. of } \\
\text { arat in } \\
\text { FLC }\end{array}$} & \multirow[t]{2}{*}{$\begin{array}{l}\text { Area of } \\
\text { FLC (dec.) }\end{array}$} & \multicolumn{2}{|c|}{$\begin{array}{l}\text { No. of fisherman } \\
\text { selling fish }\end{array}$} & \multicolumn{2}{|c|}{$\begin{array}{l}\text { Selling fish by fisherman } \\
(\%)\end{array}$} & \multicolumn{2}{|c|}{$\begin{array}{l}\text { Amount of fish selling by } \\
\text { fisherman (kg) (In hundred) }\end{array}$} \\
\hline & & & Arat/day & FLC/day & In arat & In paiker & Arat/day & FLC/day \\
\hline Dhuldiya & 11 & $26.4 \pm 1.57$ & $96 \pm 37$ & $220 \pm 37$ & $93 \pm 3.74$ & $7 \pm 3.74$ & $10 \pm 2.42$ & $44.0 \pm 6$ \\
\hline $\begin{array}{l}\text { Chamta } \\
\text { ghat }\end{array}$ & 51 & $55 \pm 1.44$ & $43 \pm 8$ & $1148 \pm 232$ & $95.56 \pm 4.44$ & $4.44 \pm 4.44$ & $6.22 \pm 2.44$ & $269.78 \pm 127.95$ \\
\hline Karimgonj & 7 & $44 \pm 4$ & $70 \pm 25$ & $173 \pm 47$ & $81 \pm 6.40$ & $19 \pm 6.40$ & $4.75 \pm 1.45$ & $26.30 \pm 7.10$ \\
\hline $\begin{array}{l}\text { Morichkha } \\
\text { li }\end{array}$ & 12 & $24.78 \pm 5.37$ & $226 \pm 11$ & $431 \pm 35$ & $98.44 \pm 0.29$ & $1.33 \pm 0.33$ & $5.05 \pm .92$ & $9.44 \pm 2.31$ \\
\hline Tarial & 25 & $29.13 \pm 3.29$ & $8 \pm 1$ & $149 \pm 27$ & $85 \pm 8.02$ & $15 \pm 8.02$ & $5.71 \pm 2.87$ & $114.64 \pm 57.36$ \\
\hline Kargaon & 12 & $11 \pm 0.87$ & $226 \pm 11$ & $431 \pm 35$ & $93.33 \pm 3.33$ & $5.83 \pm 3.27$ & $4.29 \pm .62 .72$ & $42.67 \pm 6.64$ \\
\hline $\begin{array}{l}\text { Rodar } \\
\text { poddar }\end{array}$ & 11 & $40 \pm 4.18$ & $8 \pm 1$ & $149 \pm 27$ & $97 \pm 2$ & $3 \pm 2$ & $3.33 \pm .87$ & $29.14 \pm 6.95$ \\
\hline Kotiyadi & 10 & $122 \pm 33.41$ & $76 \pm 47$ & $659 \pm 241$ & $92 \pm 5.83$ & $8 \pm 5.83$ & $8.90 \pm 2.26$ & $90.4 \pm 25.37$ \\
\hline Chouganga & 42 & $33.75 \pm 4.73$ & $63 \pm 17$ & $368 \pm 79$ & $83.75 \pm 5.54$ & $16.25 \pm 5.54$ & $3.30 \pm .92$ & $92.5 \pm 38.97$ \\
\hline Nikli & 6 & $25 \pm 8.42$ & $67 \pm 45$ & $377 \pm 136$ & $100 \pm 0$ & $0 \pm 0$ & $10.13 \pm 6.71$ & $55 \pm 15.55$ \\
\hline In total & $19 \pm 5$ & $41.11 \pm 9.77$ & $88 \pm 25$ & $410 \pm 97$ & $92.01 \pm 2.07$ & $7.99 \pm 2.08$ & $6.17 \pm .82$ & $77.39 \pm 23.85$ \\
\hline
\end{tabular}

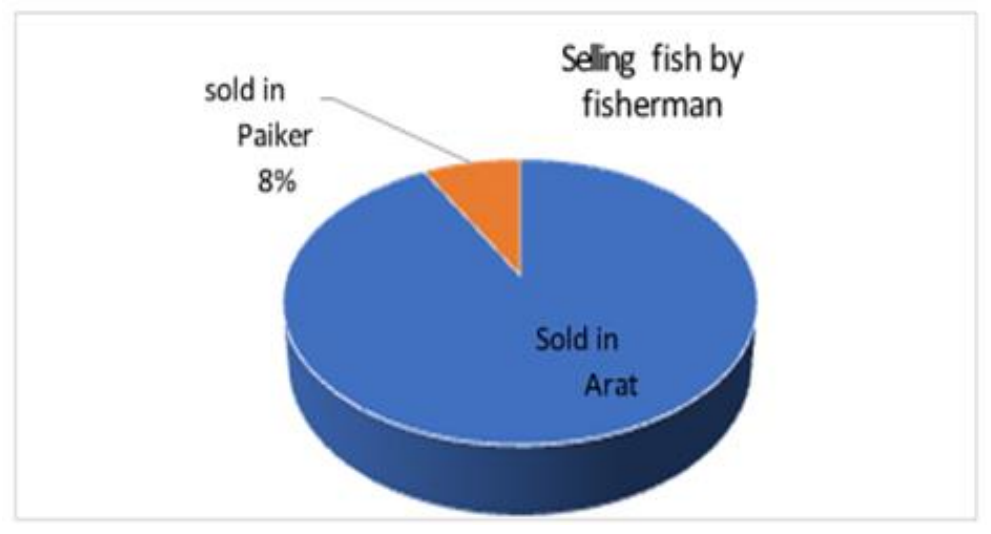

Figure 7. Status of selling fish in arat and paiker by fishermen in Kishoreganj haor. 


\section{Conclusions}

Kishoreganj haor is one of the richest sources of captured fish in Bangladesh. As understood by the study, a wide variety of fishes are available in this water body. Thousands of people are directly or indirectly depended on this water body for their livelihood. Kishoreganj haor fishes are transported to the capital and other metropolitan cities. Through this study, the species diversity, catch composition, production season, landing pattern, handling and quality conditions and transportation of Kishoreganj haor fishes have been understood. Further studies are required to explore detailed information on species biodiversity, vulnerability in landing due to overfishing fisheries, value chain analysis and improvement, etc.

\section{Acknowledgements}

The authors gratefully acknowledge Krishi Gobeshona Foundation (KGF) for funding this research (KGFBKGET/TF 38 -F/17). Thanks are due to the fishing communities in Kishoreganj haor for their supports and collaborations during the study.

\section{Conflict of interest}

None to declare.

\section{References}

Alam MK and MR Hasan, 2010. Protection works against wave attacks in the haor areas of Bangladesh: analysis of sustainability. J. Con. Dev. Coun., 15: 69-85.

Alam MS, MA Quayum and MA Islam, 2010. Crop production in the haor areas of Bangladesh: insights from farm level survey. The Agri., 8: 88-97.

Ali MY, 1997. Fish, water and people reflections on inland open water fisheries resources of Bangladesh.

BCAS, 1991. The Floodplain production monitoring. Initial Study Report. BCAS, Dhaka, Bangladesh. 99 p

CEGIS, 2012: Master plan of haor area (II), Bangladesh Haor and Wetland Development Board (BHWDB), Ministry of Water Resources, Government of the People's Republic of Bangladesh.

Chandra KJ, D Sarker, MA Khaleque and DR Das, 2010. Economic analysis of floodplain aquaculture at Daudkandi upazilla in Comilla. J. Bang. Agri. Uni., 8: 323-332.

Chowdhury MUI, 2016. Wetland-community resilience to flash flood hazards (Bonna) in Sunamganj district, Bangladesh.

DoF, 2012. National fisheries week souvenir-2012, Department of Fisheries, Government of Bangladesh, MatshayaBhaban, Dhaka. 144 pp.

DoF, 2012. National fisheries week souvenir, Department of Fisheries, Government of Bangladesh, Matshaya Bhaban, Dhaka.

DoF, 2017-18. Yearbook of fisheries statistics of Bangladesh, 35, Fisheries Resources Survey System (FRSS), Department of Fisheries, Bangladesh: Ministry of Fisheries and Livestock, 2018, p. 129.

DoF, 2019. Jatiyo matshaw saptaha sangkalan, Department of Fisheries, Bangladesh. 142 p.

DoF, 2019. Yearbook of fisheries statistics of Bangladesh, 2018-19. Fisheries Resources Survey System (FRSS), Department of Fisheries, Bangladesh : Ministry of Fisheries and Livestock, 2019. Volume 36:135 p.

FAO, 2018. The state of world fisheries and aquaculture - meeting the sustainable development goals, Italy, Rome, 2018.

Hasan M, AKMS Hasan and MS Bhuyan, 2017. Fish diversity assessment of the haor region in Kishoreganj district, Bangladesh. Res. J. Environ. Sci., 11: 29-35.

Holder DK, 2002. Studies on the availability of small indigenous species (SIS) of fishes and socioeconomic condition of fishers in two beelsof rice fields. M.S. Thesis, Department of Fisheries Management, Bangladesh Agricultural University, Mymensingh. 136 p.

Hossain MR, M Akteruzzaman, MT Parvin, MSU Bhuiya, and SMA Hossain, 2013. Marketing channel of fish and value addition by different actors in haor area of Netrokona district. Bang. J. Crop Sci., 24: 131-140.

Islam A, SS Basak and DZ Hossain, 2020. Fish and plankton biodiversity in the Kishoreganj haor, Kishoreganj, Bangladesh. Genetics of Aquatic Organisms, 4: 39-48.

Islam KA, MA Bashar and MS Ali, 2008. Diversity of fish fauna in Tanguar haor system, Bangladesh. J. Zoo., 36: 167-174.

IUCN - Bangladesh, 2004. Introduction to community based haor and floodplain resource management, IUCN Bangladesh country office, Dhaka, Bangladesh, vi+42 pp. 
Jahan R, M Quaiyum, A Sarker, BS Hossain, MB Jaman, KKB and S Rahman, 2014. Biodiversity and seasonal abundance of small indigenous fish species (SIS) in the rivers and adjacent beels of Karimganj (Kishoreganj, Bangladesh). Asian J. Ani. Sci., 8: 38-46.

Master Plan of Haor Areas, 2012. Draft final report, Vol. II, Ministry of Water Resources, Bangladesh Haor and Wetland Development Board, Government of the People's Republic of Bangladesh, pp. 1-18.

MoWR, 2010. Master Plan of Haor Areas, Bangladesh Haor and Wetland Development Board, Government of People's Republic of Bangladesh.

NERP, 1995. Specialist study wetland resources (Final report), Northeast Regional Water Management Project, FAP-6, Flood Plan Coordination Organization.

Nowreen S, SB Murshed, AS Islam, B Bhaskaran and MA Hasan, 2014. Changes of rainfall extremes around the haor basin areas of Bangladesh using multi- member ensemble RCM. Theoretical and Applied Climatology, 119: 363-377.

Nowsad AKMA, 2010. Final Report PR\# 5/08 Post-harvest Loss Reduction in Fisheries in Bangladesh: A Way Forward to Food Security, National Food Policy Capacity Strengthening Programme

Nowsad AKMA, 2016. Final report. Production enhancement of aquaculture through innovative technologies in cage culture system in haorareas of Karimganj, Kishoreganj, Project Code: TF 13-F (2013). Krishi Gobeshona Foundation.50 pp.

Nowsad AKMA, 2019. Baseline Survey Report of the KGF Project "Post-harvest loss reduction and valueaddition of freshwater fish". KGF Project: TF 38-F/2017. Submitted to Krishi Gobeshona Foundation. Farmgate Dhaka. 127 p.

Nowsad AKMA, A Shahriar, MK Rahman and MUMA Zakaria, 2020. Landing pattern of fish in open water fisheries projects of Cumilla, Bangladesh. Middle-East J. Sci. Res., 28: 439-447.

Pandit D, M Kunda, A Harun-Al-Rashid, MA Sufian and SK Mazumder, 2015. Present status of fish biodiversity in Dekhar haor, Bangladesh: a case study. World J. Fish Marine Sci., 7: 278-287.

Rahman MK and JN Akhter, 2015. Ecology and management of inland water and fisheries resources of Bangladesh. Ayub Ali Publication, 38/2 ka Banglabazar, Dhaka. 417 p.

Salauddin M and AKMS Islam, 2011. Identification of land cover changes of the haor area of Bangladesh using modis images. In: 3rd international conference on water and flood management (ICWFM- 2011).

Salauddin M and AKMS Islam, 2011. Identification of land cover changes of the haor area of Bangladesh using Modis Images. 3rd International Conference on Water and Flood Management (ICWFM-2011).

Sayeed MA, 2010. Fish biodiversity in the Chalan beel, a natural depression in North West Bangladesh. PhD Dissertation, Department of Fisheries Biology and Genetics, BAU, Mymensingh, Bangladesh. 195 p.

Sayeed MA, RC Deb, D Bhattarcharjee, MH Himu and MT Alam, 2015. Fish biodiversity of Hakaluki haor in north-east region of Bangladesh. In: Research Progress Report-2, Sylhet Agricultural University, Sylhet, Bangladesh. pp. 71-78.

Suravi IN, MS Islam, N Begum, MA Kashem, FJ Munny and F Iris, 2017. Fish bio-diversity and livelihood of fishers of Dekar haor in Sunamganj of Bangladesh. J. Asia. Soci. Bang. Sci., 43: 233-244. 